\title{
Si PIN Radiation Sensor with CMOS Readout Circuit
}

\author{
Yu-mi Kwon, Hee-Sung Kang, Jung-Hee Lee, and Yong Soo Lee ${ }^{+}$
}

\begin{abstract}
Silicon PIN diode radiation sensors and CMOS readout circuits were designed and fabricated in this study. The PIN diodes were fabricated using a $380-\mu \mathrm{m}$-thick 4 -inch $\mathrm{n}+\mathrm{Si}(111)$ wafer containing a $2-\mathrm{k} \Omega \cdot \mathrm{cm} \mathrm{n}$ - thin epitaxial layer. CMOS readout circuits employed the driving and signal processes in a radiation sensor were mixed with digital logic and analog input circuits. The primary functions of readout circuits are amplification of sensor signals and the generation of the alarm signals when radiation events occur. The radiation sensors and CMOS readout circuits were fabricated in the Institute of Semiconductor Fusion Technology (ISFT) semiconductor fabrication facilities located in Kyungpook National University. The performance of the readout circuit combined with the Si PIN diode sensor was demonstrated.
\end{abstract}

Keywords: Si PIN diode, Si epitaxy wafer, Radiation sensor, CMOS readout circuit, Fast response

\section{INTRODUCTION}

The detection of excessive radiation has attracted world-wide attention since the occurrence of nuclear plant accidents such as Japan's Fukushima nuclear crisis. Some radiation energy is very fatal and can seriously damage human organisms and/or electronic systems. Therefore, for quick detection and establishment of countermeasures against radiation incidents, a compact and portable radiation detection system used on-chip technology must be developed.

Radiation detection systems are typically used in harsh environments such as space shuttles, x-ray generators, aircrafts, weapon systems, and medical systems. Radiation sensors have been taken advantage of semiconductors as the basic materials [1-3]. Moreover, the detection efficiencies of both compound semiconductors and $\mathrm{Si}$ devices have been studied extensively [4-7]. Because compound semiconductors have variable bandgaps, diverse wavelength sensing applications are available $[8,9]$. Moreover, space charge accumulation and charge-carrier trapping that lower optical responsivity in compound

School of Electronics Engineering, Kyungpook National University 80 Daehakro,Bukgu, Daegu, 702-701,Korea

${ }^{+}$Corresponding author: leesoo@femtoline.com

(Received: Dec. 27, 2013, Accepted: Jan. 17, 2014)

This is an Open Access article distributed under the terms of the Creative Commons Attribution Non-Commercial License(http://creativecommons.org/ licenses/bync/3.0) which permits unrestricted non-commercial use, distribution, and reproduction in any medium, provided the original work is properly cited. semiconductor sensors can be mitigated by using silicon $[6,10]$. Therefore, in this study, we adopted a Si PIN diode for a radiation sensor connected to CMOS ROIC (readout circuit).

Different analog and digital readout circuit signals transferring diode signals to subsequent signal process steps have been developed. In this study, we employed a fast signal processing circuit and low-power consumption logic for the successful operation of the readout circuit. The CMOS ROICs were fabricated in ISFT CMOS fabrication facilities of Kyungpook National University.

\section{EXPERIMENTAL}

\subsection{Si PIN diode fabrication}

The fabrication process flow of a PIN diode is shown in Fig. 1. PIN diodes were fabricated using a 380-um-thick 4-inch $\mathrm{n}+$ Si(111) wafer having a resistivity of $0.004 \sim 0.008 \Omega \cdot \mathrm{cm}$ and containing a $2-\mathrm{k} \Omega \cdot \mathrm{cm} \mathrm{n}$ - thin epitaxial layer. Masks used for diode fabrication consist of LOCOS, ion implantation for $\mathrm{p}+$ and guardring regions, and contact open processes for metallization. Initially, active region isolation between devices was performed using the LOCOS process. Subsequently, boron ions were implanted in the $\mathrm{p}+$ and guard-ring regions at $50 \mathrm{KeV}$ with a dose of $1 \times 10^{15} \mathrm{~cm}^{-2}$. A 300 -nm-thick $\mathrm{SiO}_{2}$ film was subsequently deposited using TEOS to prevent the out-diffusion of implanted ions, and then, the samples were activated at $1000^{\circ} \mathrm{C}$ for $2 \mathrm{hrs}$ in $\mathrm{N}_{2}$ ambient. After the annealing process, the TEOS layers were 
selectively etched in a BOE solution to open the active regions. TiN/Al $(30 \mathrm{~nm} / 300 \mathrm{~nm})$ anodes were deposited using a metal sputtering process followed by selective etching. In addition, for the rear cathode, $\mathrm{Ti} / \mathrm{Al}(30 \mathrm{~nm} / 300 \mathrm{~nm})$ metal layers were formed with an electron-beam evaporator. Finally, an RTP process was performed to improve metal adhesion.

The device structure and photograph of the fabricated PIN diode are shown in Fig. 2. The final $2 \mathrm{~mm}$ diameter rounded shape

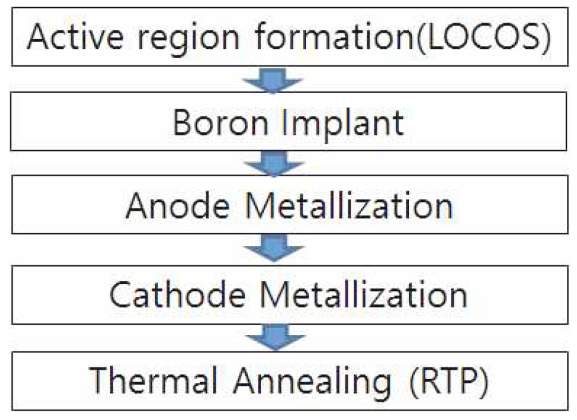

Fig. 1. Fabrication process flow of PIN diode.

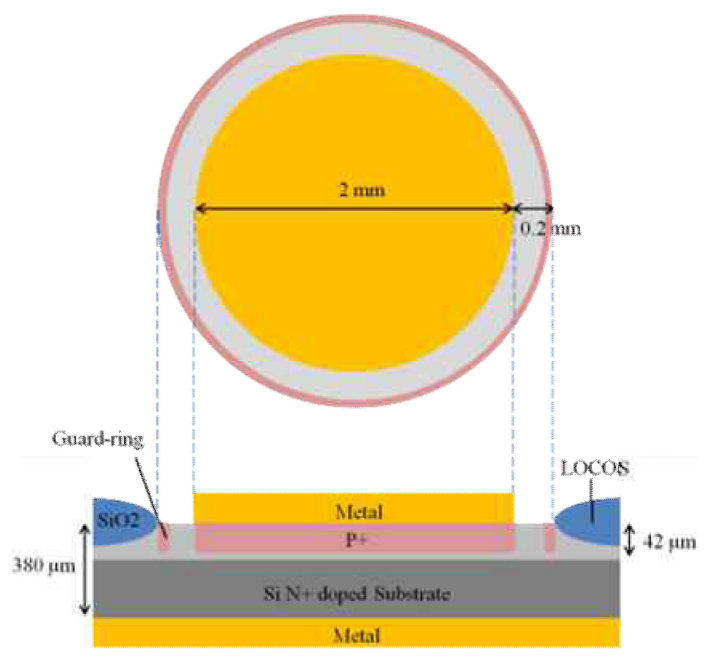

(a)

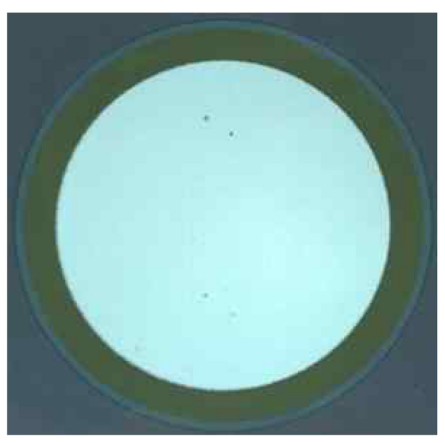

(b)

Fig. 2. (a) Structure and (b) photograph of fabricated PIN- diode. of the PIN diode was achieved several rounds of experiments. The gap between the devices namely the width of the LOCOS regions is $1 \mathrm{~mm}$ from edge of the $50-\mu \mathrm{m}$-wide guard-rings. The fabricated devices were diced with a saw blade and a laser cutting system for inspecting leakage current on the side-walls. Thereafter, the fabricated diodes were packaged in TO-cans for the long-term stabilization and measurement of radiation effects.

\subsection{CMOS readout circuits design and fabrication}

The CMOS ROIC used for driving and signal processing in the radiation sensor was mixed with digital logic and analog input circuits. The primary functions of readout circuits include sensor signal reading and warning signal or system shut down signal generation when an excessive energy radiation excess status occurs. In addition, the signal processing circuit includes a pulse timer circuit to enable the re-operation of a system after the warning signal disappears, which is an auxiliary function of the electronic system reset. A set-reset (SR) latch that provides a flag signal was used for indicating that a radiation has been sensed. The readout circuits have been referred to as a radiation-event detector (RED) in this paper.

The block diagram of the digital part of the RED readout circuit is shown in Fig. 3, and the functions of the circuit are listed in Table 1. The RED circuit comprises four inputs and several outputs. The four inputs are a radiation-events comparator output (RECO), a $1 \mathrm{MHz}$ clock (clk1M), an external reference input signal (ref), and a radiation-event flag reset (RFR). The RECO signal is generated from an open-loop comparator with inputs of the amplified sensor signal and reference voltage. The outputs include radiation event output (RO), radiation event flag (RF), and counter signals. The operation principle of the circuit is as follows. First, the RECO input signals from the output of the PIN diode

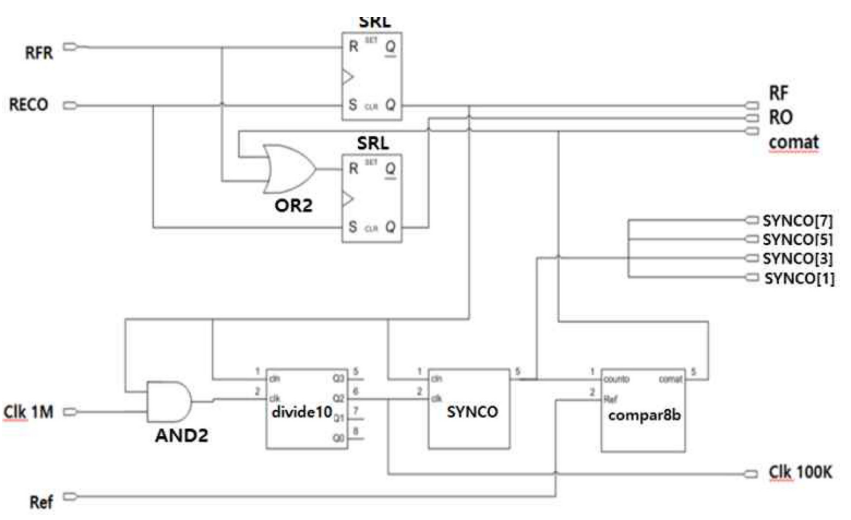

Fig. 3. Block diagram of readout circuit. 
Table 1. Description of input-output signals

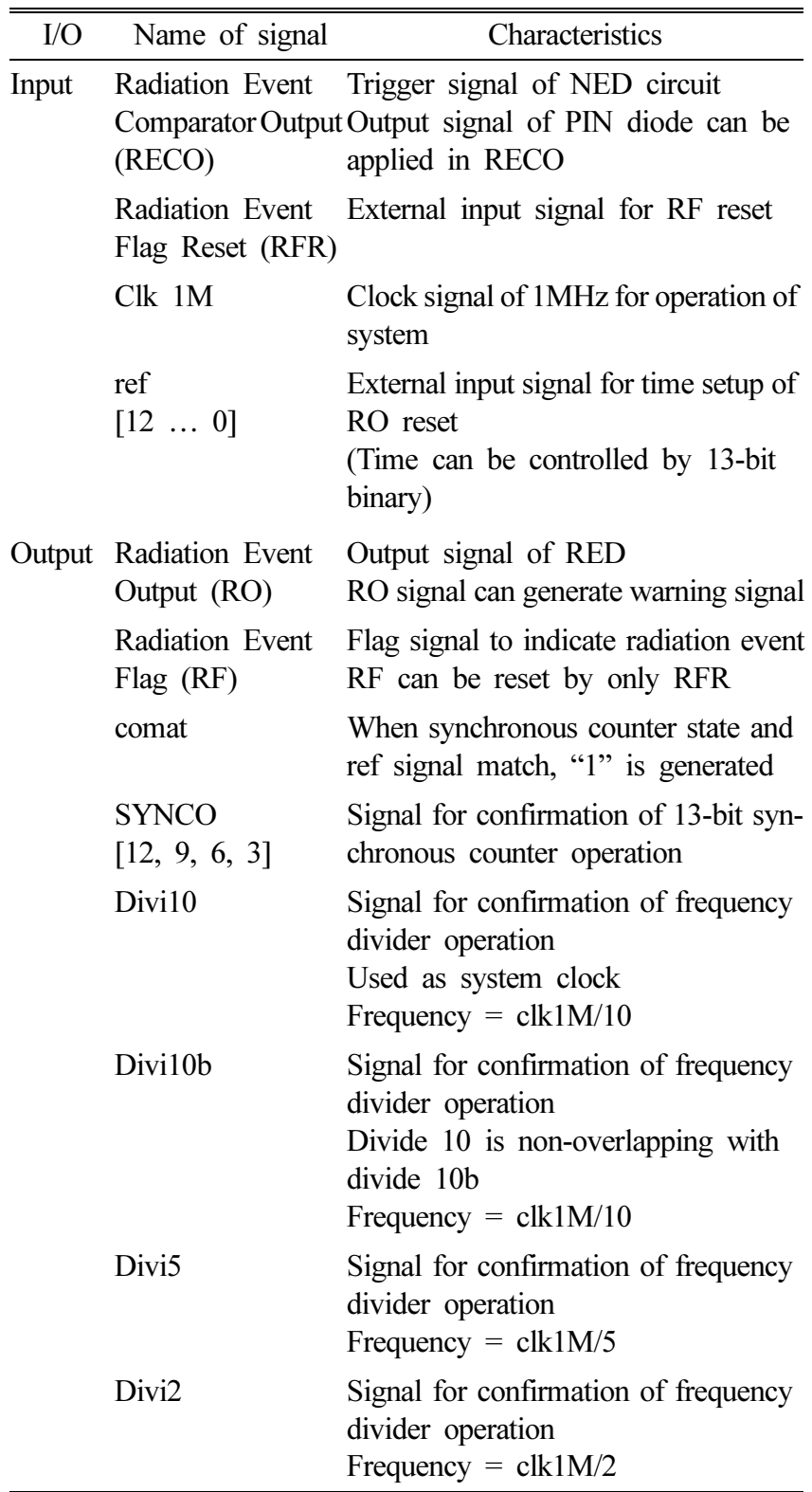

sensor enter two SR latches. If RF signal is generated, it indicates that a radiation event has occurred. If an RO signal is generated, it indicates that an excessive radiation status has occurred. Second, the RF output from the SR latch enters the AND2 gate, frequency divider (divide10), and SYNCO (13-bit synchronous counter) simultaneously. Subsequently, the AND2 gate receiving the clk1M (clock signal of $1 \mathrm{MHz}$ ) can be activated. As a result, the divide10 counter outputs the divided signals of $100 \mathrm{KHz}$, $200 \mathrm{KHz}$, and $500 \mathrm{KHz}$. Among these divided frequencies, the $100 \mathrm{KHz}$ terminal is connected to SYNCO.

The synchronous counter can count up to 8192 steps, which is an input of the comparator. When the ref input signal matches with the SYNCO output, a signal referred to as comat will be

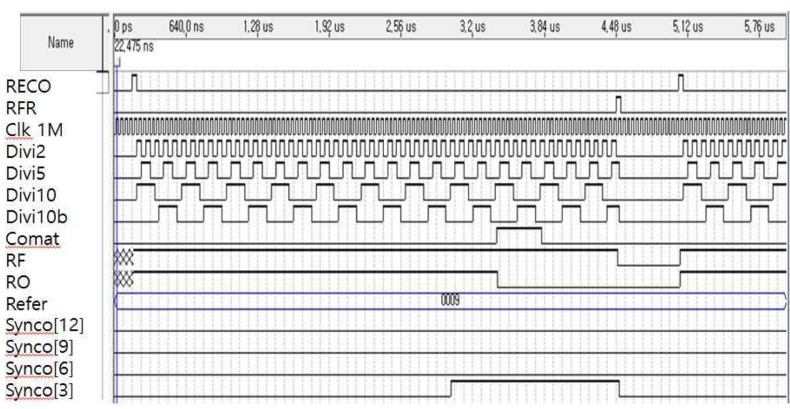

Fig. 4. Simulation results of CMOS circuit.

generated. Third, this comat signal enters the OR2 gate located in front of the SR latch generating the RO signal. As a result, the SR latch maintains the RESET and RO signals at a low level. Finally, an external RFR signal is applied for initialization, and the SR latch maintains the reset status. Further, each part of the circuit such as frequency divider, SYNCO, and comat can be monitored using the output of the operation checking procedure.

\subsection{Circuit simulation}

The simulation results of the CMOS readout circuits are shown in Fig. 4. When the RECO is produced, the RF and RO signals are immediately generated. The external clock signal of the timer clk1M is divided into divi2, divi5, divi10b, and divi10 for the control of the timer circuits. When the divi10 and the SYNCO [3] of the external reference "0009" signal match, the comat will be generated and RO enters the reset state. When an RFR occurs, all the signals are reset before the RECO signal reoccurs.

\subsection{Mask design of RED circuit}

Fig. 5 shows the mask layout of the CMOS readout circuits. The circuit consists of 10 mask layers: n-well, LOCOS, gate, $n+$ implant, $\mathrm{p}+$ implant, contact area open, $1^{\text {st }}$ metal, via open, $2^{\text {nd }}$ metal, and pad. Test patterns are prepared for analyzing of CMOS device characteristics such as resistance, capacitance, and MOSFETs. A bar-type sheet resistance measurement pattern with a length of $100 \mu \mathrm{m}$ and width of $15 \mu \mathrm{m}$ was designed. TLM pattern for the measurement of contact resistance was designed with a width of $200 \mu \mathrm{m}$ and a contact area of $45 \mu \mathrm{m}^{2}$ with a separation of $30,40,50,60$, and $70 \mu \mathrm{m}$ between contacts. The capacitance pattern was designed with a width of $365 \mu \mathrm{m}$ wide. The NMOSFET and PMOSFET of unit device were designed with a length of $2 \mu \mathrm{m}$ and width of $40 \mu \mathrm{m}$. 


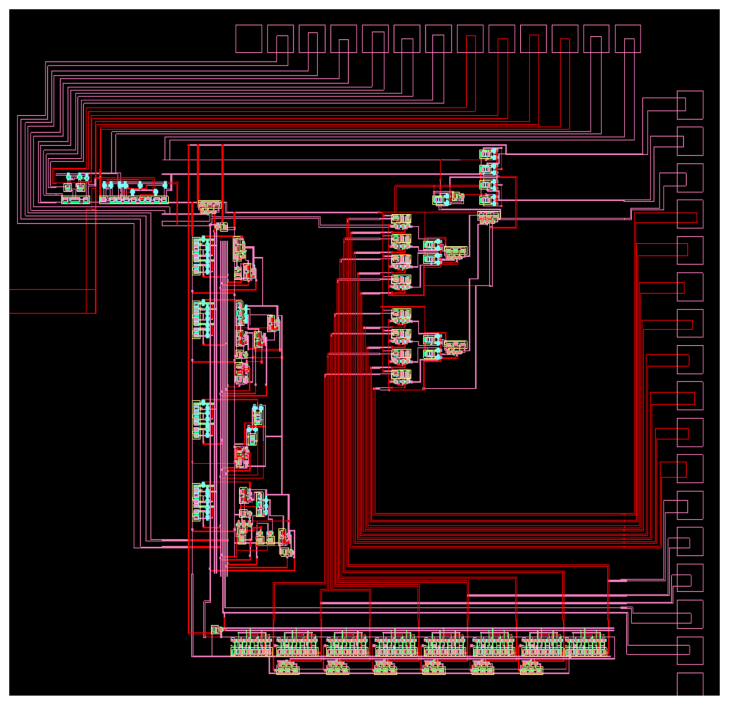

Fig. 5. Mask layout of CMOS IC.

\subsection{Fabrication of CMOS circuit}

The fabrication process flow of the CMOS readout circuits is shown in Fig. 6. The CMOS circuits were fabricated on 6-inch ptype (100) Si substrates with a thickness of $650 \mu \mathrm{m}$ and a resistivity of $0.004 \sim 0.008 \cdot \mathrm{cm}$. To fabricate the CMOS circuits, nand $\mathrm{p}$-well regions were formed by injecting phosphorus and boron ions. After activation of the implanted ions followed by annealing, LOCOSs having an oxide thickness of $5200 \AA$ were grown outside the active region for isolating each device. Thereafter, phosphorus ions were implanted in MOSFET channels for controlling work function. Thin $250 \mathrm{~nm}$ gate oxides and $200 \mathrm{~nm}$ phosphorus-doped polysilicon gates were stacked sequentially on the MOSFET gate area for n-type formation. An inductively coupled plasma (ICP) etching process was used for gate patterning in polysilicon, and arsenic and boron ions were implanted for source and drain formation by implantation, respectively. Subsequently, a 300-nm-thick-oxide was grown using a tetraethyl orthosilicate (TEOS) process for contact open. E-beam evaporated Ti/TiN ( $40 \mathrm{~nm} / 800 \mathrm{~nm})$ at a metal/Si contact area was used for silicide formation using rapid thermal processing (RTP) at $900^{\circ} \mathrm{C}$ for $5 \mathrm{~min}$ in $\mathrm{N}_{2}$ ambient. The $1^{\text {st }}$ metallization with $\mathrm{Ti} / \mathrm{Al} / \mathrm{TiN} \quad(10 \mathrm{~nm} / 300 \mathrm{~nm} / 80 \mathrm{~nm})$ was performed immediately after silicide formation. A 500-nm- thick oxide was deposited again on $1^{\text {st }}$ metal surface for $2^{\text {nd }}$ metallization. Finally, after via open, the $2^{\text {nd }}$ metal of Ti/Al/TiN $(10 \mathrm{~nm} / 450 \mathrm{~nm} / 80 \mathrm{~nm})$ was deposited and patterned finally. After fabrication, the readout circuits were separated by sawing and packaged on a PCB using a wire bonder.

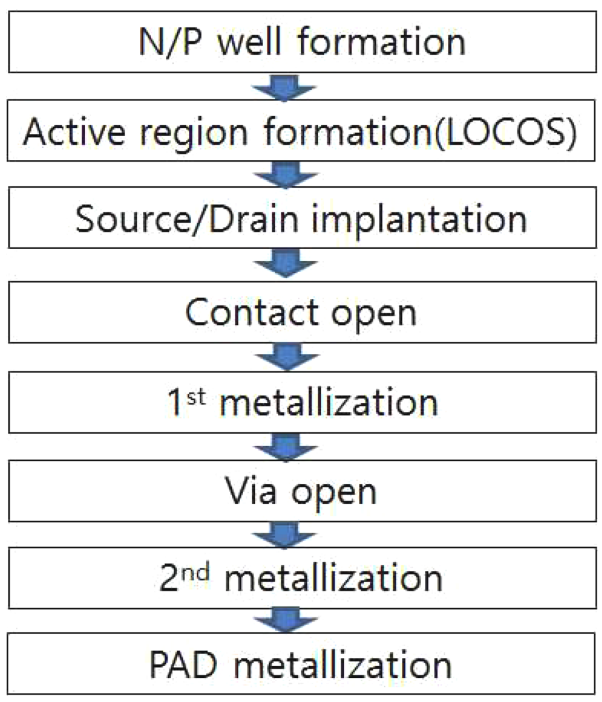

Fig. 6. CMOS readout circuit fabrication flow.

\subsection{Measurement of readout circuit}

A field programmable gate array (FPGA) that generates driving pulses of CMOS readout circuit was used for the characterization of readout circuit. The RFR, clk1M, and RECO having a pulse width of 80 ns pulse were applied from the FPGA to the readout circuit operated at a bias voltage of $3 \mathrm{~V}$. The RECO for the verification of operation was generated $3 \mu$ s after RFR generation was used. The value of ref supplied from the timer circuit was fixed to be " 1001 ". The outputs of the RF and RO were generated through an inverter, which can compensate for the voltage drop. When the circuit is connected to the PIN diode, FPGA can be replaced with RECO. The anode of the PIN diode was connected to $72 \Omega \mathrm{k}$ and $10 \Omega \mathrm{k}$ parallel resistors. The output of the PIN diode can be acquired at the resistor nodes. This output can be connected to the RECO input terminal of the circuit. Moreover, the RFR, clk1M, and ref, which were the inputs of the circuit, were applied by the FPGA.

Each component of circuit, such as the frequency divider and the synchronous counter was verified by checking the output of the comat signal.

\subsection{Measurement of electrical characteristics of PIN diode}

The electrical characteristics of the fabricated PIN diodes were measured in the dark at room temperature. The top of the diode anode in direct contacted with probe tip, and rear of the diode cathode was connected through aluminum paste and plate. The 


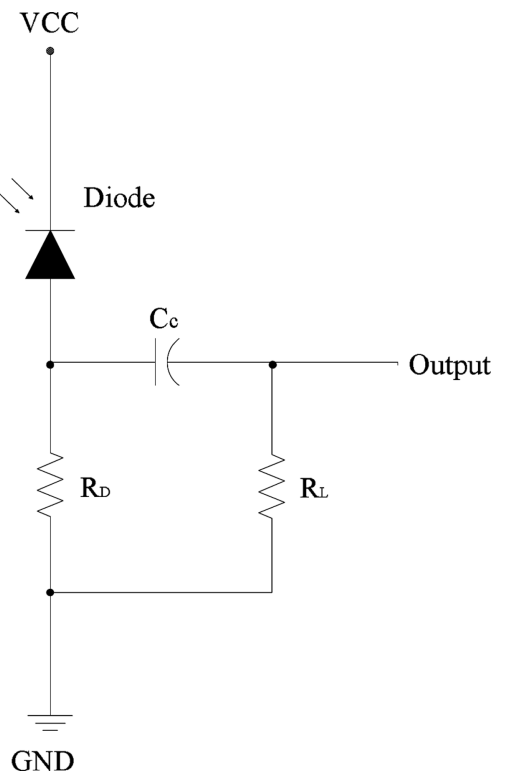

Fig. 7. Output circuit configuration of PIN diode.

current-voltage (I-V) characteristics were measured with HP-4156 observable a photo-electric current in the reverse bias state. HP4284 was employed for the obtaining of reverse biased diode capacitance and a fully depleted voltage deducible from $\mathrm{C}-\mathrm{V}$ characteristics. Because the capacitance significantly delays diodes response, the smallest possible depletion capacitance diode must be used.

\subsection{Measurement of radiation response characteristics}

Radiation response characteristics of the fabricated diodes were investigated by using a simple circuit which consists of the diode, a bias resistance $\left(R_{D}\right)$ of 50 , load resistance $\left(R_{L}\right)$ of $1 M$, and a coupling capacitor $\left(\mathrm{C}_{\mathrm{C}}\right)$ of $10 \mathrm{nF}$, as shown in Fig. 7. The diode biased at a reverse voltage of $10 \mathrm{~V}$ was irradiated, and its radiation incident response was investigated.

\section{RESULTS}

\subsection{Current-voltage characteristics}

Fig. 8 shows the I-V characteristics of the fabricated PIN diode when the applied forward-bias voltage was varied from 0 to $2 \mathrm{~V}$, and the reverse-bias voltage varied from 0 to $-30 \mathrm{~V}$. In case of forward-bias voltage, the current increased exponentially as voltage increased. The forward voltage $\left(\mathrm{V}_{\mathrm{F}}\right)$ of the fabricated diode was 0.6 V. Further, reverse leakage current (dark current)

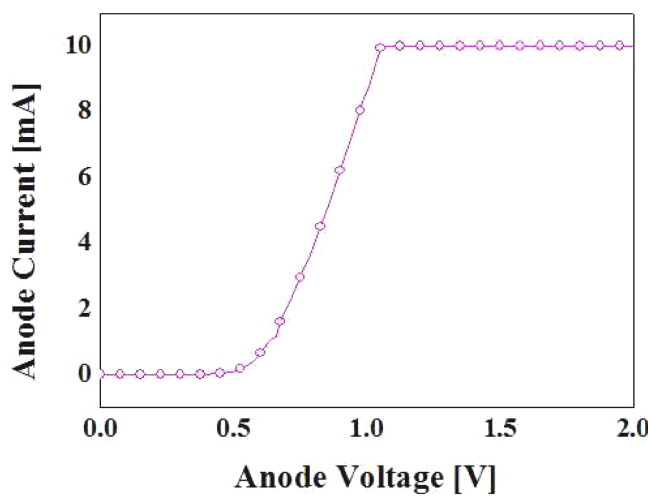

(a)

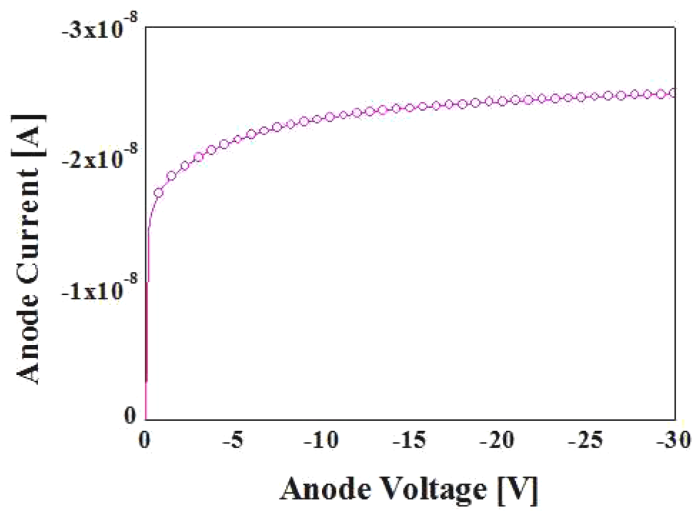

(b)

Fig. 8. C-V characteristics of PIN diode with (a) forward- and (b) reverse-bias voltage.

low is an important for the generation of enough photon current because it can be a noise source when the radiation is incident to the PIN diode. The fabricated PIN diode exhibited an extremely low leakage current of $24 \mathrm{nA}$ at a reverse bias voltage of $30 \mathrm{~V}$ owing to the surface passivation through the LOCOS process and the $\mathrm{p}+$ guard-ring located in the outer part of the device, which can interrupt electron flow from the $\mathrm{n}+$ bulk region to surface of the diced wafer.

\subsection{Capacitance-voltage results}

Fig. 9 shows (a) the capacitance-voltage and (b) depletion characteristic of the PIN diode. Since the depletion capacitance in the reversed-biased operation influences on the response speed largely due to resistance capacitance (RC) delay, the capacitance must be investigated to confirm the feasibility of the fabricated PIN diode. The decreased depletion capacitance caused by the reverse-bias voltage increase is shown in Fig. 9. Measured capacitance was enough low value of $12.5 \mathrm{pF}$ at a bias voltage of $-30 \mathrm{~V}$. Low full depletion voltage acquired here is appropriate 


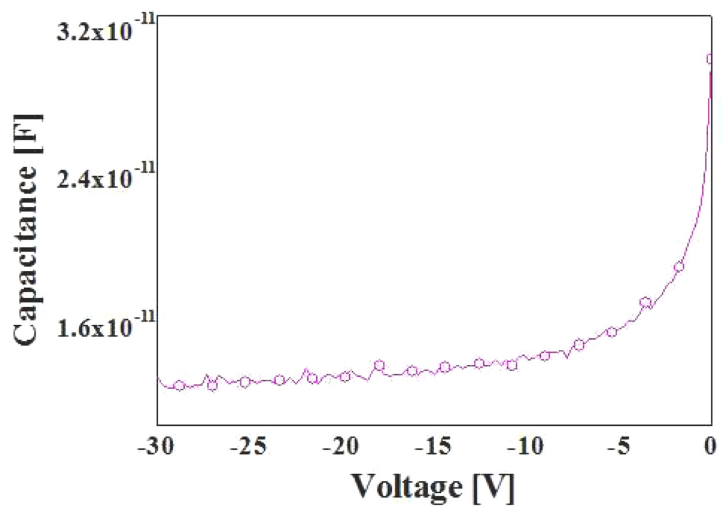

(a)

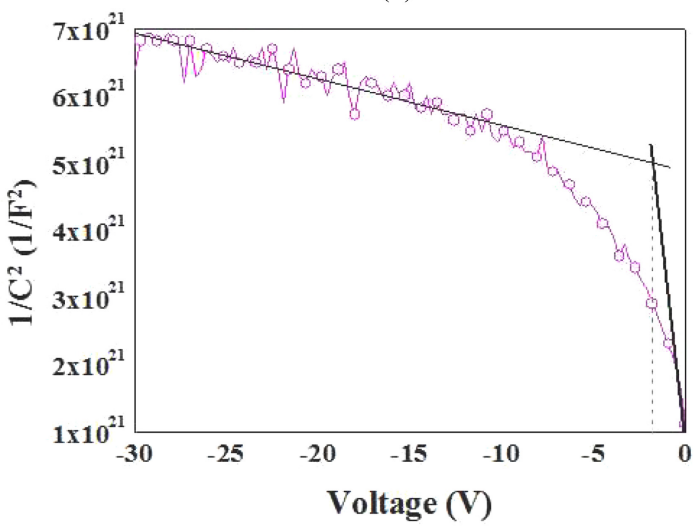

(b)

Fig. 9. (a) Capacitance-voltage and (b) full-depletion voltage characteristics of PIN diode.

value to ensure the stable operation of the PIN diode as radiation sensor.

\subsection{Response to radiation}

The photocurrent of the PIN diode generated by pulse gammaray irradiation at an output node was investigated. Peak voltage detection method was used at load resistance. The delay time measured between input and output signal was measured to be $1.51 \mu \mathrm{s}$. This delay time is not considered due to the RC delay of the fabricated diode, but considered due to the delayed electron beam generation in the system. The rising time between $10 \%$ and $90 \%$ of maximum output voltage, was approximately $201.8 \mathrm{~ns}$. This relatively slow rising time is considered to be caused by the $\mathrm{RC}$ delay originating from passive components such as $\mathrm{R}_{\mathrm{D}}$ and $\mathrm{C}_{\mathrm{C}}$. This delay is not related to the diode characteristics because the fabricated diode has an extremely low capacitance. In addition, the acquired output voltage of $7.43 \mathrm{~V}$ is sufficient for high driving in readout circuits.

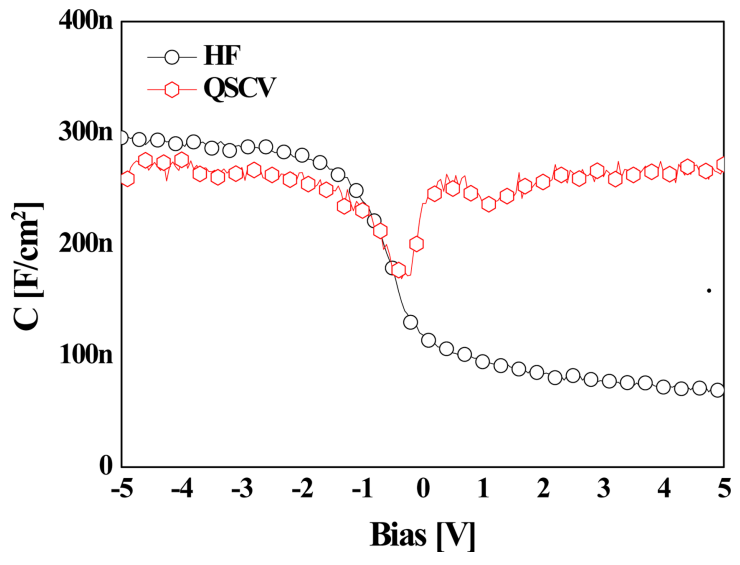

Fig. 10. Capacitance-voltage characteristics of gate insulator.

\subsection{Experimental results of readout circuit}

The electrical characteristics of the test patterns such as TLM, capacitor, NMOSFET, and PMOSFET were measured in a shielded test-box using HP-4156 and HP-4284. The sheet resistances of source/drain in each $\mathrm{n}+$ and $\mathrm{p}+$ regions were $100 \Omega / \square$ and $94 \Omega / \square$ evaluated from the TLM and capacitance measurements. The measured sheet resistance of the $n+$ poly silicon was approximately $300 \Omega / \square$, which was greater than expected.

The contact resistance of the Ti-silicide was also calculated using TLM measurement. The contact resistance adapted silicide process was reduced by one-tenth from $8 \times 10^{-3} \Omega \cdot \mathrm{cm}^{2}$ to $1.1 \times 10^{-4}$ $\Omega \cdot \mathrm{cm}^{2}$ after the silicide process. However, the calculated sheet resistance, in the $\mathrm{n}+$ and $\mathrm{p}+$ of source/drain regions and the poly silicon region were 63,126 , and $272 \Omega / \square$, respectively, and were similar to the sheet resistance values. In the case of contact resistance, each value was changed to $1.9 \times 10^{-3} \Omega \cdot \mathrm{cm}^{2}, 2.3 \times 10^{-4}$ $\Omega \cdot \mathrm{cm}^{2}$ and $1.1 \times 10^{-4} \Omega \cdot \mathrm{cm}^{2}$, respectively. Moreover, the contact resistance of the poly silicon was lower than that of the silicon substrate, because the silicide forms easily between the metal and poly silicon. On the other hand, the general contact resistance was higher than that in the conventional CMOS process. To improve the contact resistance, the activation temperature and period for the silicide formation must be investigated.

Fig. 10 shows the capacitance-voltage characteristics of gate insulator which was measured using $100 \mathrm{KHz}$ high frequency and quasi static $\mathrm{CV}$ (QSCV) methods traceable $\mathrm{SiO}_{2}$ gate insulator thickness and defects at $\mathrm{SiO}_{2}$ and $\mathrm{Si}$ interface. Measured capacitance was $300 \mathrm{nF} / \mathrm{cm}^{2}$ at the gate voltage of $-5 \mathrm{~V}$. The calculated thickness of the $\mathrm{SiO}_{2}$ by $\mathrm{C}-\mathrm{V}$ plot and the measured thickness by ellipsometer were similarly $11.6 \mathrm{~nm}$ and $12.5 \mathrm{~nm}$. The 


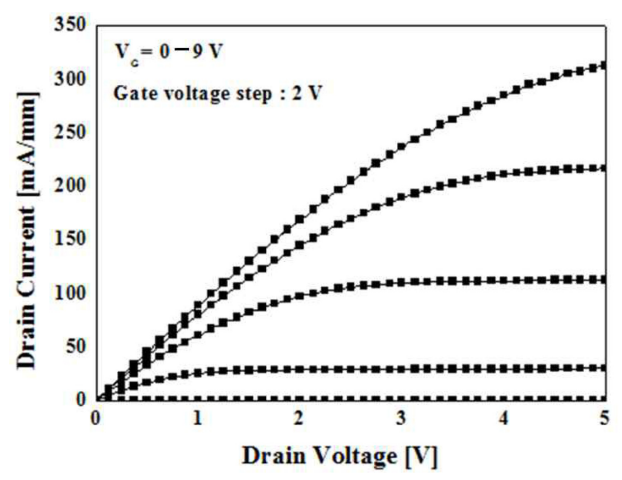

(a)

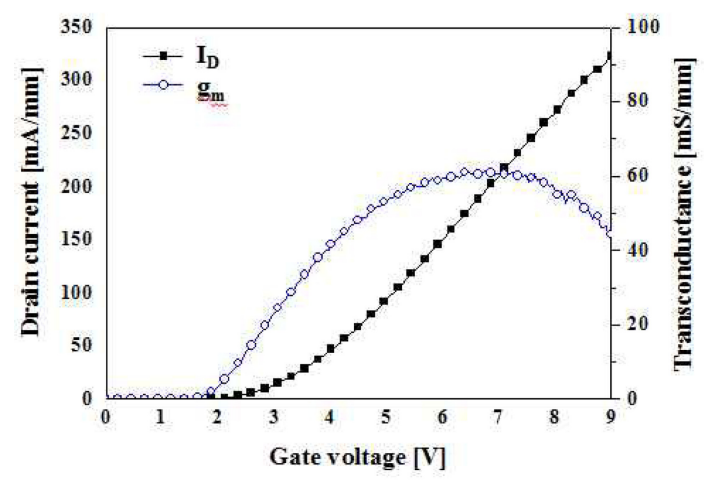

(b)

Fig. 11. DC characteristics of NMOSFET unit device.

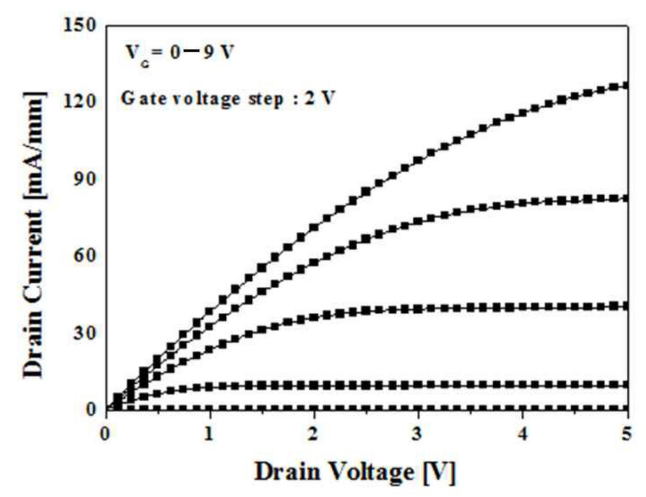

(a)

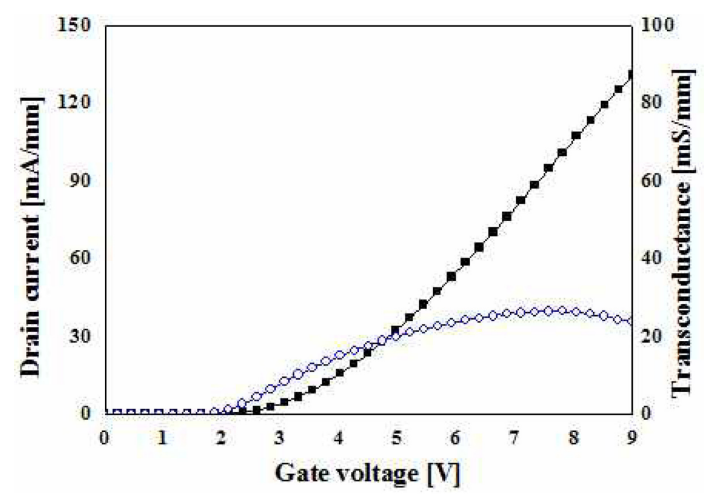

(b)

Fig. 12. DC characteristics of PMOSFET unit device. interface state density was calculated to be $3.61 \times 10^{-11} \mathrm{~cm}^{-2} \mathrm{eV}^{-1}$ and threshold voltage $\left(\mathrm{V}_{\mathrm{T}}\right)$ was to be $0.5 \sim 0.8 \mathrm{~V}$.

The DC characteristics of the NMOSFET unit device are shown in Fig. 11. High maximum drain-source current $\left(\mathrm{I}_{\mathrm{DMAX}}\right)$ of $335 \mathrm{~mA} / \mathrm{mm}^{2}$ at $\mathrm{V}_{\mathrm{G}}=9 \mathrm{~V}$ was obtained. The I-V characteristics of Fig. 11(b) show that the threshold voltage is $1.6 \mathrm{~V}$ higher than that of calculated value from $\mathrm{C}-\mathrm{V}$ curves. The $\mathrm{DC}$ characteristics of the PMOSFET unit device are shown in Fig. 12. $\mathrm{I}_{\mathrm{DMAX}}$ of the PMOSFET is also high at $126 \mathrm{~mA} / \mathrm{mm}^{2}$, and the threshold voltage is shown $-1.9 \mathrm{~V}$.

The field-effect mobility of the NMOSFET and PMOSFET were obtained in the linear region at $\mathrm{V}_{\mathrm{ds}}=0.1 \mathrm{~V}$. The maximum field effect mobility of the both the devices were $185 \mathrm{~cm}^{2} / \mathrm{Vs}$ and $83.6 \mathrm{~cm}^{2} / \mathrm{Vs}$, respectively. The low field mobility is typically required to eliminate the effect of the parasitic series resistance and the high interface state density. The gate-leakage characteristics of the two unit devices showed pico-scale values. Thus, the gate insulator which had the low leakage current was satisfactory for the fabrication of the CMOS readout circuit fabrication.

\subsection{Measurement of circuit}

Fig. 13 is outputs at each node of frequency dividers, which are used for confirming the feasibility of the fabricated CMOS readout circuits. The main system clock (clk1M) of the $1 \mathrm{MHz}$ pulse input is shown on the top in Fig. 13. The measured clk100k and inverted state of clk100k showed precise $10 \mu$ s durations. The clk $200 \mathrm{k}$ with a $5 \mu$ s periods and clk $500 \mathrm{k}$ with a $2 \mu$ s per cycle are resulted in Fig. 13. From this well functioned frequency divider composed of CMOS circuits, the operation of the RED readout circuits fabricated in the ISFT semiconductor facility can be guaranteed.

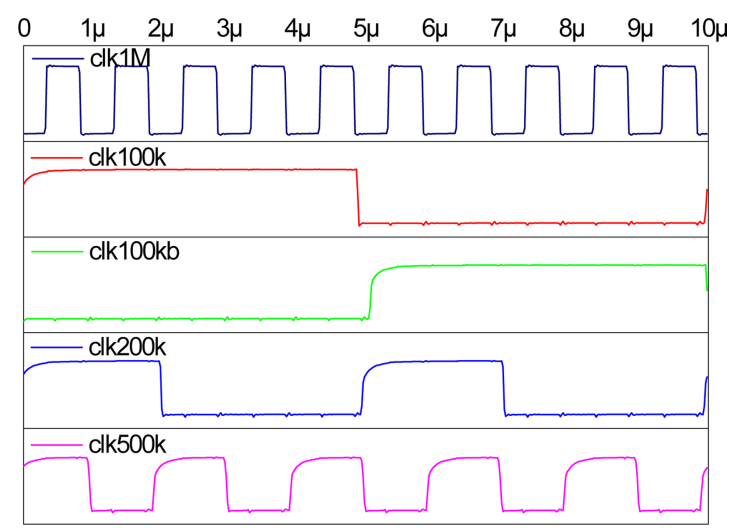

Fig. 13. Output characteristics of frequency divider. 


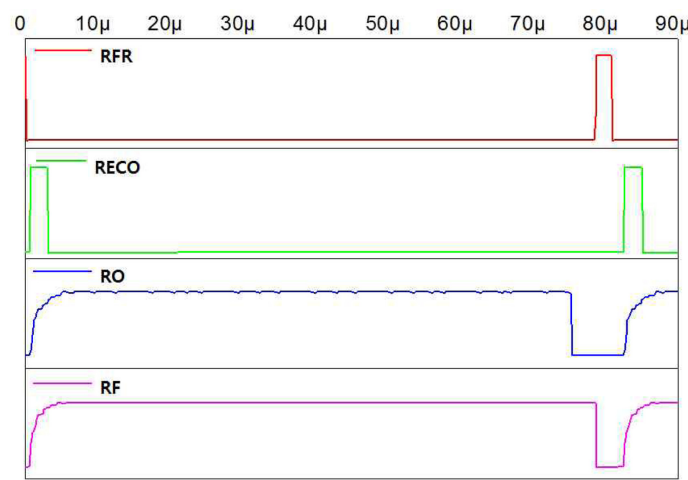

(a)

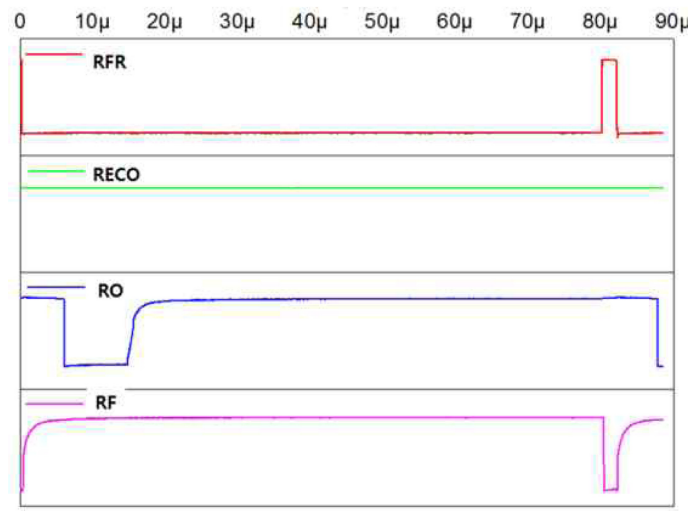

(b)

Fig. 14. Signal response of readout circuits (a) without and (b) with Si PIN diode.

Fig. 14 shows the response of the readout circuits. Fig. 14 (a) shows the response of the readout circuits without connection to the Si PIN diode, and Fig. 14(b) shows the combined response of the Si PIN diode and the CMOS readout circuits. In Fig. 14(a), the RFR input signal was applied at intervals of $80 \mu \mathrm{s}$, and the RECO supplied by the FPGA was applied $3 \mu$ s after the RFR. When the RECO was applied, the maximum output signals of RO and RF was generated in $3 \mu$ s through SR latches. In addition, RO was owing to a fixed " 1001 " reference counter signal generated after $75 \mu$ s. When the RFR signal was applied, the RO signal was maintained until the comat signal generated, as shown in the figure. Fig. 18(b) shows the signal output of the RED circuits connected with the PIN diode. In this case, an LED was used in place of high energy gamma-ray irradiation. The output voltage of the PIN diode was approximately $3 \mathrm{~V}$ once the LED irradiated the PIN diode. The RECO signal was constant at a high level as long as the LED was irradiating the RED. When the RECO was applied, a rising time of $700 \mathrm{~ns}$ was observed in the RO signal response.

\section{CONCLUSION}

This research focused on the fabrication of a Si PIN diode as a radiation detector and the implementation of a readout circuit with the detector. The readout circuits were designed for the generation of radiation alarms when a certain amount of radiation incidents occur. Further, by adding a signal processing circuit, the total amount of radiation rate could be evaluated.

The Si PIN diode was fabricated with a Si epitaxial wafer with a maintainable low full-depletion voltage because of comparably thin epitaxial layer. The thickness of epitaxial layer was optimized to minimize the variation in performance. Thus, the PIN diode could be operated stably when exposed to high energy radiation incidents.

The readout circuit connected to the Si PIN diode generated processing signals, which were then sequentially transferred to the readout circuit, sequentially. In conclusion, the feasibility of onchip radiation detectors containing a silicon readout circuit, which was fabricated in the ISFT facility of Kyungpook National University, is demonstrated.

\section{ACKNOWLEDGMENT}

This study was supported by the National Research Foundation of Korea (NRF-2010-0025679) and by Korea Atomic Energy Institute (UC11 0026ID). The authors are deeply grateful for this support.

\section{REFERENCES}

[1] Y. I. Kim, H. J. Hyun, D. H. Kah, H. D. Kang, H. J. Kim, H. O. Kim, and H. Park, "Radiation damage study of a silicon PIN diode and signal-to-noise ratio mearsurement with a proton beam", J. Korean Phys. Soc., vol. 54, pp. 20662070, 2009.

[2] G. Lutz, Semiconductor Radiation Detectors, Springer Verlag, Berlin, 1999.

[3] S. Glasstone and P. J. Dolan, The Effects of Nuclear Weapons $3^{\text {rd }}$ edn, US GPO, Washington, D. C. 1977.

[4] S. Jha, Emil V. Jelenkoviæ, M. M. Pejoviæ, G. S. Ristiæ, M. Pejoviæ, K. Y. Tong, C. Surya, I. Bello, and W. j. Zhang, "Stability of submicron AlGaN/GaN HEMT devices irradiated by gamma rays", Microelectronic Engineering, vol. 86, pp. 37-40, 2009.

[5] J. S. Iwanczyk, B. E Patt, Y. J Wang, and A. Kh Khusainov, "Comparison of $\mathrm{HgI}_{2}, \mathrm{CdTe}$ and $\mathrm{Si}$ (p-i-n) X-ray detectors", Nucl. Instr. Meth. Phys. Res., vol. A380, pp. 186-192, 1996.

[6] D. S. McGregor and H. Hermon, "Room-temperature com- 
pound semiconductor radiation detectors", Nucl. Instr. Meth. Phys. Res., vol. A 395, p. 111, 1997.

[7] J. H. Park, S. H. Seo, I. S. Wang, J. K. Shin, and P. Choi, "Highly sensitive active pixel sensor using a PMOSFET photodetector", J. Korean Phys, Soc. vol. 44, p. 157, 2004.

[8] A. Owens and A. Peacock, "Compound semiconductor radiation detectors", Nucl. Instr. Meth. Phys. Res., vol. A 531, pp. 18-37, 2004.
[9] K. S. Shah, J. C. Lund, F. Olschner, L. Moy, and M. R. Squillante, "Thallium bromide radiation detectors", IEEE Trans. Nucl. Sci., vol. 36, pp. 199-202, 1989.

[10] R. Wunstorf, W. M. Bugg, J. Walter, F. W. Garber, and D. Larson, "Investigations of donor and acceptor removal and long term annealing in silicon with different boron/phosphorus ratios", Nucl. Instr. Meth. Phys. Res., vol. 14, p. 109, 2000 . 